\title{
A Novel Call Admission Control Policy Using Mobility Prediction and Throttle Mechanism for Supporting QoS in Wireless Cellular Networks
}

\author{
Chen-Feng Wu, ${ }^{1}$ Liang-Teh Lee, ${ }^{2}$ Hung-Yuan Chang, ${ }^{2,3}$ and Der-Fu Tao ${ }^{3}$ \\ ${ }^{1}$ Department of Information Management, Yu Da University, Miao-Li 36143, Taiwan \\ ${ }^{2}$ Department of Computer Science and Engineering, Tatung University, Taipei 10452, Taiwan \\ ${ }^{3}$ Department of Electronic Engineering, Technology and Science Institute of Northern Taiwan, Taipei 11202, Taiwan
}

Correspondence should be addressed to Chen-Feng Wu, cfwu@ydu.edu.tw

Received 11 April 2011; Revised 11 July 2011; Accepted 3 August 2011

Academic Editor: Ricardo Dunia

Copyright (c) 2011 Chen-Feng Wu et al. This is an open access article distributed under the Creative Commons Attribution License, which permits unrestricted use, distribution, and reproduction in any medium, provided the original work is properly cited.

\begin{abstract}
The decision of call admission becomes an important work owing to the scarce wireless spectrum for wireless cellular networks. If there exists adequate information for call admission control (CAC) schemes, the terms of quality of service (QoS), such as call dropping probability (CDP), call blocking probability (CBP), and system utilization, will be kept in a certain acceptable level. Therefore, a prediction system which can predict most information, such as system utilization and CDP, in advance with a novel data mining technique and a distributed CAC scheme is presented in this paper. Based on the prediction results and the bandwidth consumption of adjacent cells, the proposed CAC scheme is able to decide to admit a new call. The throttle flag that can indicate the usage of current cell is proposed to prevent the newly admitted call request from being blocked in adjacent cells if handoff is needed. The simulation results show that the proposed CAC scheme can maintain the CDP below a predefined threshold, and the $\mathrm{CBP}$ is also lower than the cluster prediction and traditional guard channel policies.
\end{abstract}

\section{Introduction}

With the evolution of mobile communication, the types of services have been evolved from voice service to multimedia $[1,2]$. As a large number of bandwidths are required for multimedia services for wireless cellular networks, the most important issue is to support QoS for subscribers. Although there has been a rapid development in wireless cellular communications, the QoS guarantee remains one of the most challenging issues $[3,4]$. One of the key elements for providing QoS guarantees is an effective CAC policy, which not only has to ensure that the network meets the QoS of the newly arriving calls, but also guarantees that the QoS of the existing calls will not be degraded. Because of the increase in mobile velocity and limited radio spectra, it is difficult to allocate suitable bandwidths for mobiles before handoff to the appropriate cell for a bandwidth reservation and CAC policy. The variable user mobility has made it more complex to predict the appropriate cell for handoff. The prior researches [5-8] have showed the impact of mobility on wireless cellular networks and provided a modeling method for configuring wireless cellular networks to study the dynamics of mobility.

However, the CBP should be also mentioned simultaneously while the QoS is considered for an effective CAC policy. There have been so many studies, such as $[4,9,10]$ which had focused on the issues of QoS guarantee. Furthermore, the effective user mobility and resource management were also mentioned in [11]. The improvement of radio bandwidth is always regarded as a dynamic channel (code) allocation problem in [12]. Although there had been some schemes proposed for bandwidth reservation to reduce the CDP, such as [13], the seldom literature has been developed to guarantee the QoS and to satisfy lower CBP at the same time. Besides, the past researches mentioned above focused on individual mobility prediction, and they may incur mass of loads concentrating on the MSC (mobile switch center).

According to [11], the final information is a prediction of users' number in a given cell, and it leads to use a global approach that only observes variations of system 
utilization and users' flows. There are many advantages for the scheme presented in [11]: it does not require any control message and additional load for the MSC, cells generate their own statistics independently from others, and it is sensitive to geographical constrains and to users' common habits. Besides, the concept of aggregated history has been also applied to acquire user profile of mobility in [14] so that a profile framework of user mobility is developed for estimating service patterns and tracking mobile users' information which includes descriptions of location, mobility, and service requirements.

Therefore, based on the concept of cell approach and aggregated histories, an agent that consists of a prediction module and a CAC algorithm for a given cell was proposed in our preliminary research [15], and preliminary research are revised, extended, and verified more accurately in this paper. The proposed agent can perform the prediction and CAC works for each cell to avoid extreme computations focused on the MSC, and the global statistics can be gathered via fixed high-speed links in a periodic time. The prediction module that is based on time series prediction can provide the appropriate information for CAC algorithm to make the decision of call admission. Comparing to the traditional data mining process for time series prediction scheme, the proposed prediction method reduces calculation overheads and storage requirements significantly. Besides, throttle mechanism can be combined to reduce the CDP further, and the extra exchanged information among neighboring cells also can be reduced by exchanging throttle flags if needed. Furthermore, the proposed CAC scheme can meet the predefined network QoS with the proposed throttling mechanism and achieve an acceptable CBP.

\section{Related Works}

A wireless cellular network cannot always meet the QoS requirements of various multimedia services for mobiles owing to restricted resources. Therefore, it is difficult to admit the most appropriate call request into the system to avoid unwanted call blocking and handoff dropping while maximizing channel utilization. A suitable prediction mechanism can help the CAC scheme to make a better decision for call admission. Thus, the integration of CAC and prediction will reduce the CDP effectively to meet the QoS requirement.

2.1. Time Series Prediction. Both statistical and artificial approaches are usually applied to most of the time series prediction techniques. One of the typical statistic models that are based on statistical concepts and principles is Box-Jenkins forecasting model [16]. Although a wide spectrum of time series behavior can be modeled by such models, they cannot model well when there are noises in the time series, such as inaccurate or incomplete data.

In $[17,18]$, neural networks that are one of well-known artificial intelligent techniques had been claimed to be able to achieve better prediction compared to statistical models.
Whatever, [19] shows that there exist some drawbacks for neural networks, such as being hard to preselect the system architecture, spending much training time, and lacking facilities of knowledge representation. Besides, [20] applies the concept of data mining to perform time series prediction. Both adaptive association rules and pattern matching techniques were employed by [20] to mine for repetitive patterns, which can be applied to understand the underlying utilization of system utilization, in the time series. Although this model can perform lifelong adaptation and reinforcement learning for continuous prediction, the disadvantage is the requirement which needs intensive computation power and huge storage.

In this paper, we were devoted to develop a prediction method, having the abilities of the adaptation for dynamic wireless cellular networks, providing prediction values continuously whether periodic patterns exits or not, adjusting the confident level of patterns for lifelong prediction process, and requiring less computation power and storage. The bases of performing prediction for the proposed prediction method are both time series prediction submodule and compensation prediction submodule in this paper.

2.2. CAC Overviews. In general, handoff calls are assigned higher priority over new calls, so there have been many studies that gave preferential treatment to handoff call, such as [5]. Many different admission control strategies have been discussed in the literature to provide priorities to handoff requests without significantly forgoing new connection requests. These strategies fall into two categories: handoff queue (HQ) [21], and guard channel (GC) [22] schemes.

Nevertheless, CAC schemes can be also classified into centralized ones that make the decision of call admission in the MSC and distributed ones that make the decision of call admission in individual cell locally, and most of prior researches $[13,23,24]$ that were based on different theories and focused on the developments of centralized CAC schemes. However, there will be too many traffic loads that are call admission requests from each cell concentrating on the MSC so that the MSC cannot deal properly with each call admission request instantaneously.

Instead of using centralized CAC schemes, distributed CAC schemes have been proposed in [25-27]. In [26], the estimation information of CDP and CBP is applied to the decision of call admission. The call admission decision is made locally, and the related information is exchanged among neighboring cells for resource reservation and call admission. However, too much related information exchanged among neighboring cells will cause heavy loads and affect the efficiency of call admission. Based on the local information, Zhang et al. [27] had applied two prediction mechanisms that were historic data and time series prediction to a CAC scheme in noncollaborative manner. Unfortunately, the overheads that are caused by predicting the appropriate information for making CAC decision still exist. A clustering prediction scheme that is based on data mining concept for user mobility prediction has been proposed in [25], but the related information from neighboring cells is still required 
to participate in mobility prediction and more computation loads and storage for data mining process are also required.

For reserving the appropriate amount of resources, Heikkonen et al. [17] proposed an analytical model to estimate the resource requirements for handoff calls, and Agrawal et al. [28] proposed a dynamic scheme of resource allocation based on the estimation of maximum expected resource requirement which was needed for handoff calls. However, the above proposed schemes are either not efficient in resource allocations or time consuming in estimating the actual allocation resources.

Thus, a CAC scheme that is integrated with a time series prediction for providing effective call admissions with heuristic estimation of aggregated mobility in handoff call needs is proposed in this paper.

\section{Methodology}

For a given cell, two kinds of prediction information must be provided for a call admission control scheme for wireless cellular networks. According to two kinds of predictions, the proposed CAC can decide to admit a new call or not for preventing call drop in the future. Because of the individual characterization of time series for each cell, the concept of agent which executes the prediction, and call admission control tasks for a given cell is proposed for simplifying the implementations.

\subsection{Design Concepts of the Proposed Prediction Method. The} process flow of the proposed prediction model, as shown in Figure 1, consists of four submodules. The proposed module is a part of an agent which is built for each cell and is executed for supplying prediction information to the CAC algorithm. The prediction system obtains the environment information from the MSC periodically, or the base station controller of each cell provides its information to the prediction system. The time series data is stored in the storage of each cell and exchanged via the MSC, if needed. The proposed model can also perform other resource predictions in the same way.

3.1.1. Submodule for Time Series Data Analysis. The analysis submodule is responsible for performing data preprocessing and data mining on time series data. The purpose of data preprocessing is to convert the time series data into the gradient code which is defined by the application characteristics of prediction and available system storage. More storage is required if the gradient code is defined in a small scale. The data mining is to generate chain code pattern, and the learning results are stored into the database which will be used for the prediction task. The time series data is represented in a chain code format to keep track of the rate of change of time series. The rate-of-change of a time series is obtained by calculating the difference between each two temporal point. The gradient code is defined by using the difference to divide a predefined base which can be decided upon the prediction sensitivity and system storage. To restore the prediction code, the reminder of division is recorded as a compensation value. The purpose of transferring the difference into the gradient code is to make the exact difference of two temporal points to be a fuzzy value that represents a varying degree between two temporal points. The process flow for generating the gradient codes is illustrated in Figure 2. The time series data is represented by a function of $X(t)$, where $t$ is the current time. The difference between $X(t)$ and $X(t-1)$ is represented as $D(t)$, and the compensation value is defined as $C(t)$, where the predefined base is 10 . Besides, the division should be modified to keep the reminder being positive.

The following phase of the analysis submodule is to perform data mining for detecting the periodicity of patterns and other relative information. The process of data mining is based on the following equation to find an iterative pattern.

$$
R(d)=\operatorname{set}\left(\sum_{t=i}^{i+n-1}|G(t+d)-G(t)|\right)
$$

The function set in (1) decides whether a repetitive pattern exists or not. If there exists a repetitive pattern of length $n$, which represents the size of pattern window, then $n$ differences between two gradient vectors must be all zero with distance $d$, where $d$ is the delay $(d \geq n)$ and $i$ is the time of the first point in the preceding pattern. Patterns with different distances between the pattern length and the delay are managed in different lists. The occurrence times of periodic patterns and the average of compensation values will be recorded in the database.

3.1.2. Submodule for Time Series Prediction. After data mining, the prediction submodule will make use of periodic patterns to predict possible time series data in future. If a segment of a repetitive time series pattern matches with the inputs, it may happen that the following segment of the particular repetitive pattern has the possibility of reoccurrence. However, there will be not only one kind of patterns with the same prefixes in the database, and several possible results that are based on the counts of pattern occurrence will be provided by the prediction submodule. The prediction result with the highest probability which is decided by counts of these patterns will be adopted. Because the prediction value is only a gradient code, the final output should be adjusted by the average compensation value and decoded.

Figure 3 illustrates the process for performing the prediction task. There are four periodic patterns recorded in the database. The item count records the occurrences of each periodic pattern, and these patterns appeared more than once before time $t$. In order to restore the prediction gradient code to time series data accurately, the average compensation value is recorded in the compensation list.

3.1.3. Submodule for Compensation Prediction. To provide more accurate prediction information, the compensation prediction that is based on the following equation to perform prediction generates different prediction values to supply selection submodule for choosing the most appropriate one:

$$
\begin{gathered}
C P(t+1)=\alpha \times\left(C P(t)+\overline{\varepsilon_{t}}\right)+(1-\alpha) \times X(t), \\
\overline{\varepsilon_{t}}=\frac{1}{t} \sum_{i=1}^{t} \varepsilon_{i}, \\
\varepsilon_{i}=X(i)-\tilde{X}(i) .
\end{gathered}
$$




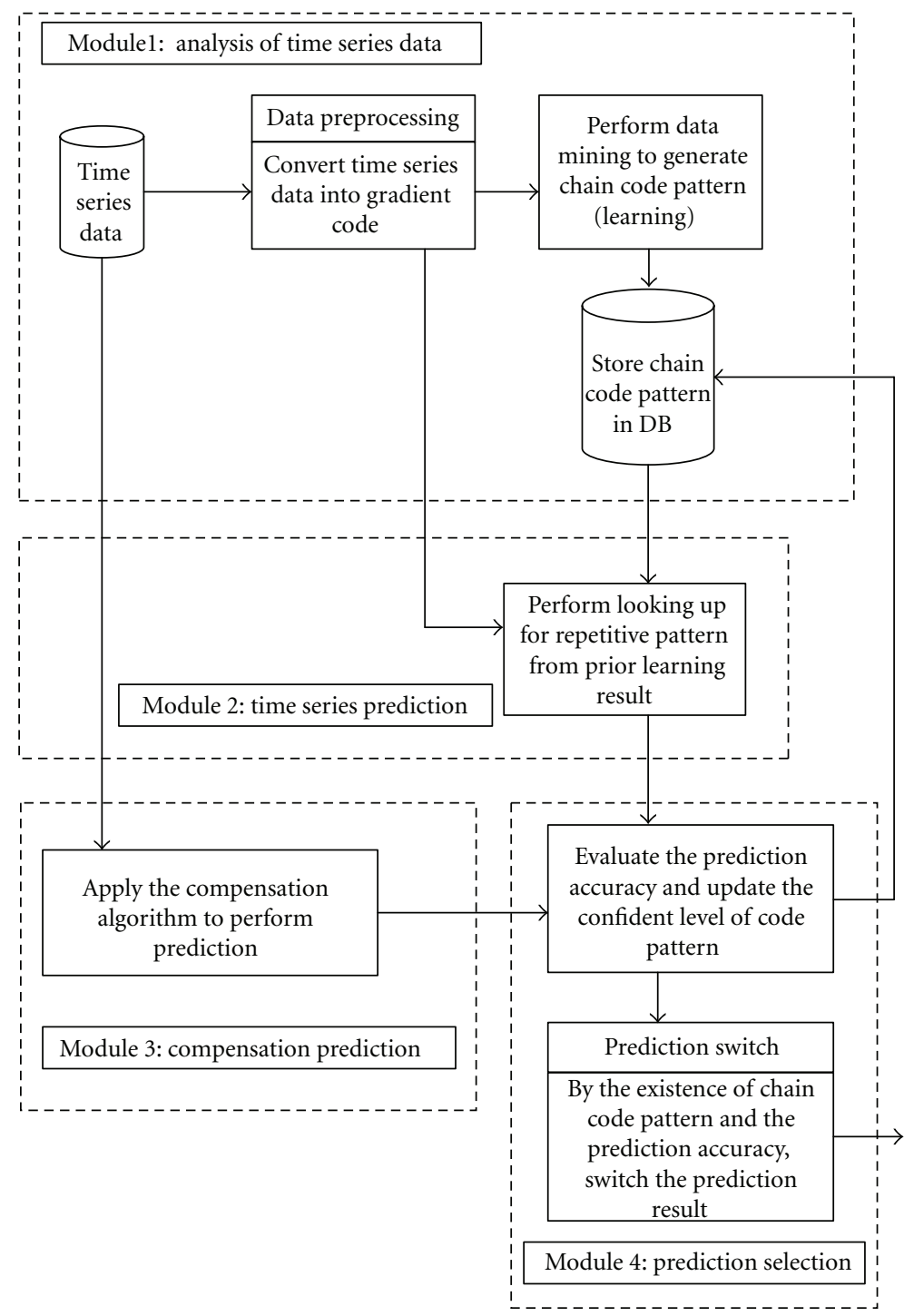

FIGURE 1: The process flow diagram of the proposed prediction method.

\begin{tabular}{|c|c|c|c|}
\hline \multirow{2}{*}{ Time series data } & \multirow{6}{*}{$\begin{aligned} \quad \text { Differencing } \\
D(1)=X(1)-X(0)=3 \\
D(2)=X(2)-X(1)=-18 \\
D(3)=X(3)-X(2)=40 \\
D(4)=X(4)-X(3)=-27\end{aligned}$} & \multirow{6}{*}{$\begin{array}{l}\text { Gradient code } \\
\begin{array}{l}G(1)=0 \\
G(2)=-2 \\
G(3)=4 \\
G(4)=-3\end{array}\end{array}$} & \multirow{6}{*}{$\begin{array}{l}\text { Compensation } \\
C(1)=3 \\
C(2)=2 \\
C(3)=0 \\
C(4)=3\end{array}$} \\
\hline & & & \\
\hline $\begin{array}{l}X(0)=30 \\
X(1)=33\end{array}$ & & & \\
\hline $\begin{array}{l}X(1)=33 \\
X(2)=15\end{array}$ & & & \\
\hline$X(2)=15$ & & & \\
\hline $\begin{array}{l}X(3)=63 \\
X(4)=38\end{array}$ & & & \\
\hline
\end{tabular}

FIgure 2: An example of generating gradient code.

For (2), the compensation prediction is concerned with the actual time series data $X(t)$ at current time $t$ and the previous prediction result $C P(t)$ at time $t-1$. The parameter $\alpha$ is used to decide the weight of the value $C P(t)$, and $\overline{\varepsilon_{t}}$ is the average difference that is the distance from actual value $X(i)$ to prediction value $\widetilde{X}(i)$ and is defined by (3). If a greater $\alpha$ is chosen it means that the preceding prediction has greater effect than the last actual value. Equation (5) which is derived from (2) with a fixed constant $\alpha$ shows that the earlier value has less effect on the prediction result:

$$
\begin{aligned}
C P(t+1)= & \alpha \times\left(C P(t)+\overline{\varepsilon_{t}}\right)+(1-\alpha) \times X(t) \\
= & \alpha^{2} \times C P(t-1)+\alpha^{2} \overline{\varepsilon_{t-1}}+\alpha(1-\alpha) \\
& \times X(t-1)+\alpha \overline{\varepsilon_{t}}+(1-\alpha) \times X(t) \\
= & \alpha^{t}(1-\alpha) \times X(0)+\cdots+\alpha(1-\alpha) \times X(t-1) \\
& +(1-\alpha) \times X(t)+\left(\alpha^{t} \overline{\varepsilon_{1}}+\cdots+\alpha^{2} \times \overline{\varepsilon_{t-1}}\right. \\
& \left.+\alpha^{1} \times \overline{\varepsilon_{t}}\right) .
\end{aligned}
$$




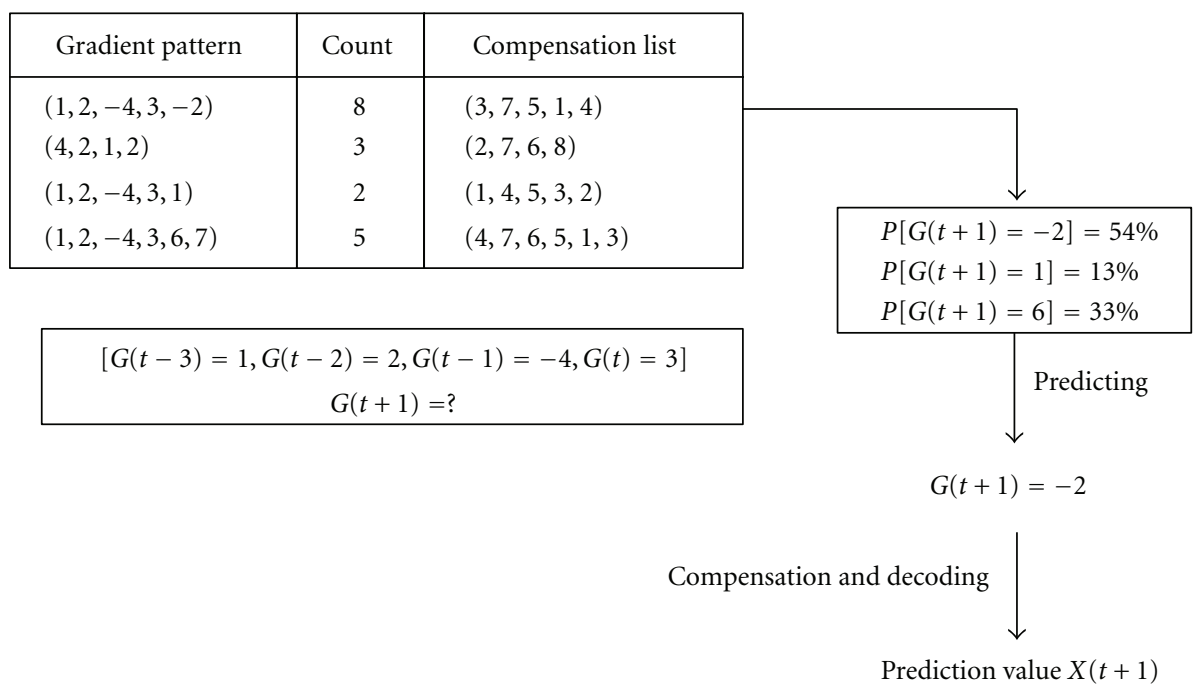

FIgURE 3: An example of performing prediction.

Furthermore, the compensation method may be more dynamic by adjusting the value for the long term process.

\subsubsection{Submodule for Prediction Selection. This submodule} is responsible for determining the final prediction result between the time series prediction and the compensation prediction and then updating the confident levels of pattern lists that is regarded as an indicator to decide whether a pattern list should be kept in the database or not. To keep an accurate prediction, the distances between predicted values and actual values should be calculated when the actual values have been received. For the prediction selection, each predicted values, $X(t+1), X(t+2), X(t+3)$, and so forth, are also recorded. Because of the continuous participations of new inputs for time series data, the proposed method is able to dynamically remind the confidence level of pattern lists as time goes on. Thus, the pattern lists with low confidence level will be gradually neglected.

The process of updating the pattern list is illustrated in Figure 4 . At time $t+1$, the actual time series data $X(t+1)$ that is with former pattern $[1,2,4,-3]$ is received and transferred into $G(t+1)$ by preprocessing process. If the actual value of $G(t+1)$ is $-2,1$, or 6 , the count value of the existing pattern list will increase, and the corresponding confident level will be raised at next prediction. A new pattern will be added to the pattern list if $G(t+1)$ is not an expected value, such as 8 . After occurring several times for this pattern, the submodule will check the delay and record the pattern to be another referable pattern in the time series prediction.

There are two defined thresholds, distance threshold and count threshold, dominating the accuracy for the time series prediction. If the difference between the prediction value and the actual value is larger than the distance threshold, the prediction will be regarded as a prediction miss. The time series prediction will be regarded as inaccuracy when the number of continuous prediction misses is more than the count threshold.
The prediction selection submodule dynamically adopts one of the prediction results that are from compensation prediction or time series prediction. For the submodule of compensation prediction, the compensation algorithm generates several results with various coefficients, $\alpha s$, and the best prediction result will be chosen according to the average distance. If there has not been any periodic pattern found or the accuracy of the time series prediction is too low, then the best prediction value that is generated by the compensation prediction will be regarded as the output. Otherwise, the prediction result of the time series patterns will be selected and sent out, and the prediction process is also completed simultaneously by the proposed prediction system.

For reducing the consumption of computation power and the storage demand, some techniques are applied to the implementation. A list that records the possible pattern length is maintained in the database. The pattern lengths with the higher occurrence probability will earlier become the window size for performing pattern detection, and it will avoid running the detecting algorithm for every single length pattern. All of the system average values are computed by (6) instead of (7):

$$
\begin{gathered}
\operatorname{Avg}(n)=\frac{n-1}{n} \times \operatorname{Avg}(n-1)+\frac{1}{n} \times \operatorname{value}(n), \\
\operatorname{Avg}(n)=\frac{1}{n} \times \sum_{i=1}^{n} \operatorname{value}(i) .
\end{gathered}
$$

where $\operatorname{Avg}(n)$ is the average of $n$ number of values, and $\operatorname{value}(n)$ is the last value in data stream. Equation (7) needs to record all of the $n$ values in database; on the contrary, (6) reduces the storage demand and less computation is needed.

3.2. The Proposed CAC Scheme. The admission of a new call request is more sophisticated than the admission of a handoff call request, because it will affect the CDP deeply. Therefore, the proposed CAC scheme, named time series prediction call 


\begin{tabular}{|l|l|}
\hline Pattern & Count \\
$(1,2,-4,3,-2)$ & 8 \\
$(4,2,1,2)$ & 3 \\
$(1,2,-4,3,1)$ & 2 \\
$(1,2,-4,3,6,7)$ & 5 \\
\hline
\end{tabular}

\begin{tabular}{|c|c|}
\hline Pattern & \\
\hline & \\
\hline$(1,2,-4,3,-2)$ & 9 \\
\hline$(4,2,1,2)$ & 3 \\
\hline$(1,2,-4,3,1)$ & 2 \\
\hline$(1,2,-4,3,6,7)$ & 5 \\
\hline
\end{tabular}
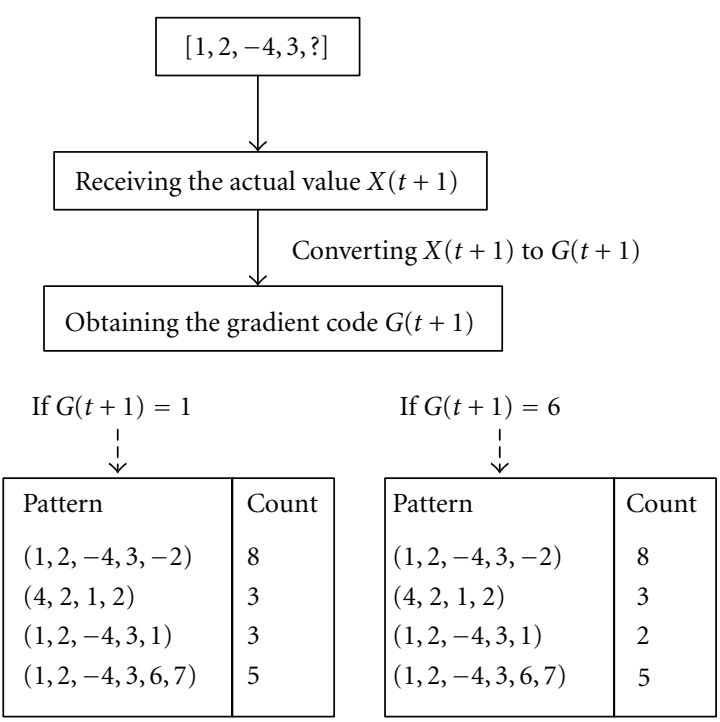

Inserting new pattern list
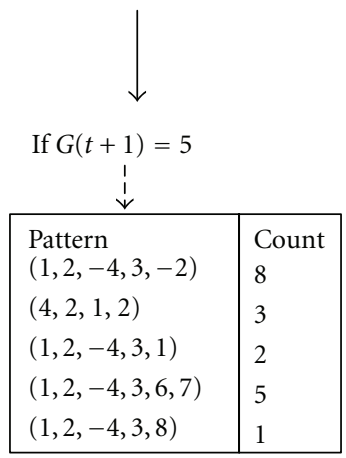

Figure 4: An example of updating the pattern list.

admission control (TSPCAC), adopts the result of time series prediction to be one of the criteria for admitting a new call request. The other criterion is the situation of bandwidth consumption for the six adjacent cells, because it will be concerned with the successful handoff probability for the newly admitted call request if a handoff is needed. Besides, the concept for maintaining the CDP below a predefined threshold, which can be decided by user demand, is also combined with the proposed CAC, and the details of the proposed CAC scheme are illustrated in Algorithm 1.

The proposed CAC can deal properly with two traffic classes; one is multimedia that requires four units of bandwidth, and the other is normal data or voice that requires one unit of bandwidth. The required bandwidth can be adjusted according to the actual demand of traffic. First, according to current system utilization and system capacity, the remaining bandwidths can be calculated for each cell. The handoff call request will be admitted if the remaining bandwidths are enough for the demand of handoff call request.

The predicted information of system utilization and CDP in the next time is provided by the proposed prediction module for the proposed CAC scheme to deal with a new call request. The throttle concept participates in deciding whether to admit a new call request or not for avoiding the new call request being blocked in the adjacent cells. When there are not enough bandwidths to be allocated to a new call request in current time, the throttling flagi for current cell $i$ will be set 1 to represent that there is no more bandwidth left. Besides, it also alerts the six adjacent cells to throttle a new call request for preventing handoff call from dropping in the future. By the same token, throttling_flagi,j, the throttle flags of the six adjacent cells of current cell $i$, are also checked for admitting a new call request when there exist enough bandwidths in current cell. Based on throttling flags, the decision which rejects a new call will depend on all, partial, or one of the throttling flags, throttling_flagi,j, has been set to 1 .
If one of the throttle flags throttling_flagi,j is set to 1 , the new call request will be rejected for the proposed CAC scheme. Because the QoS factor of CDP is regarded as the most important issue for the proposed CAC scheme, a new call request will be rejected if there is any call dropping possibility occurring when a handoff occurs for the newly admitted call. According to the individual handoff history, the new call may be properly admitted even though one or more throttling flags throttling_flagi,j are set to 1 . However, the individual handoff histories for mobiles are not adopted in this paper owing to the large storage required for maintaining individual handoff histories.

A predefined threshold thresholdCDP which can be set according to the actual demand is regarded as a criterion for admitting a new call request to keep the CDP below a certain level. The new call will be admitted when the remaining bandwidths in the future are more than four units and the CDP in the future will be below the thresholdCDP. The reason why the remaining bandwidths in the future should be more than four units is to guarantee that a handoff call request of multimedia traffic can be admitted in the future.

\section{Performance Evaluation}

Both system utilization and CDP are the aggregated history of mobiles for a given cell, and they will be predicted by the prediction module to provide the call admission information for the CAC module. There will be two evaluated tasks for simulations, and they are the performance evaluations for the proposed CAC without throttle mechanism applied and the evaluations of throttle mechanism for the proposed CAC scheme, respectively.

4.1. Evaluations of the Proposed CAC. Both CDP and CBP of QoS terms are evaluated for the proposed CAC scheme that applies the prediction results of system utilization and CDP. 


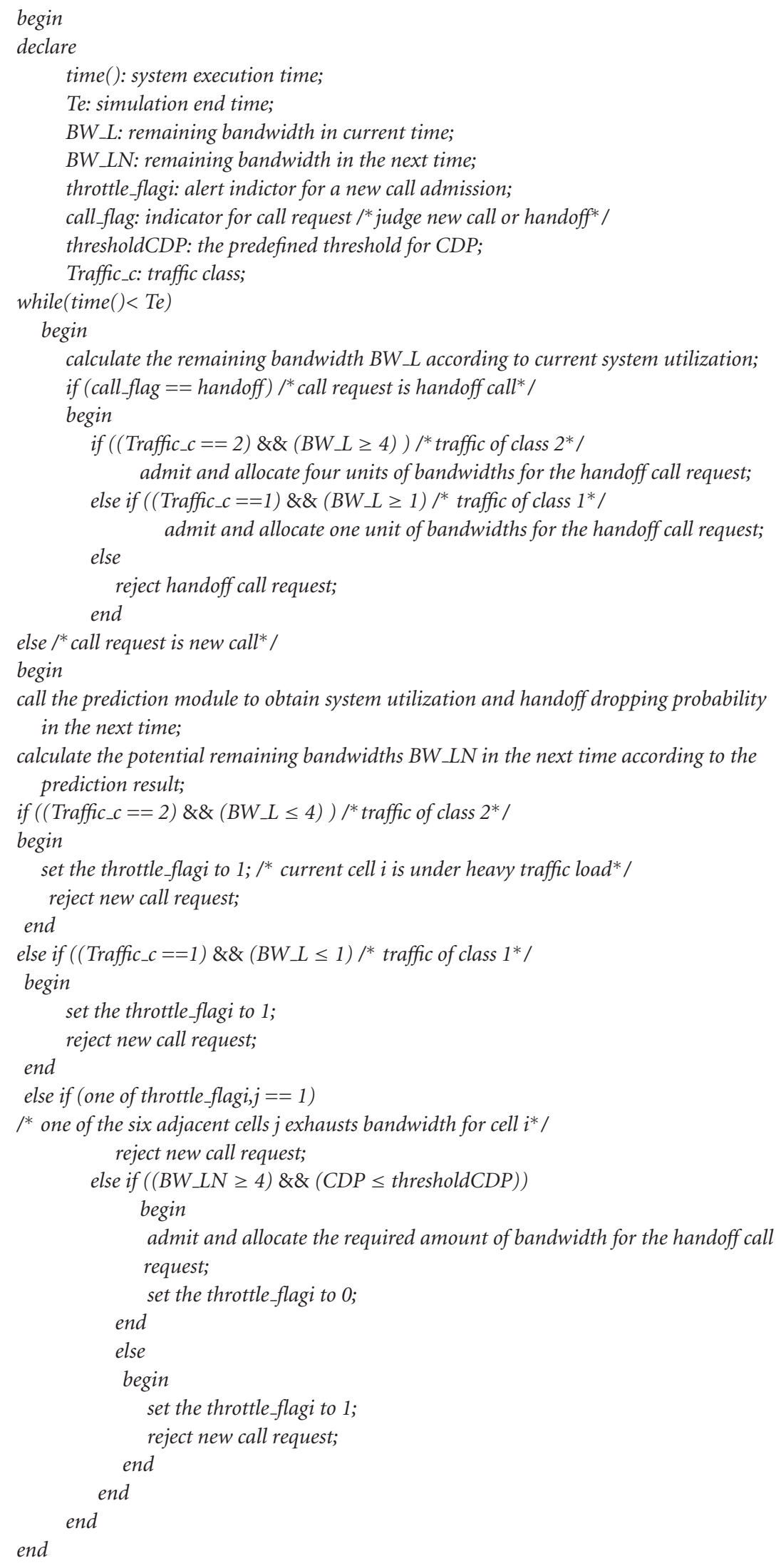

Algorithm 1: TSPCAC (time series prediction call admission control). 
The simulations are executed under a given cell that has its own time series data in system utilization and CDP, and other assumptions are described as follows.

(i) There are 20 bandwidth units for a given cell.

(ii) The traffic arrival that consists of new call and handoff call requests follows Poisson process with rate 1.

(iii) The service time follows exponential time distribution and the mean time is 6 time units.

(iv) The occurrence possibilities of handoff call and new call are 0.5 and 0.5 , respectively.

(v) There are two traffic classes; one is multimedia call request that requires 4 bandwidth units, and the other is normal voice or data call request that requires only 1 bandwidth unit.

(vi) The predefined CDP threshold thresholdCDP is 0.3 .

In order to evaluate the performance, the similar prediction scheme in [25] that adopted data mining and time series techniques called clustering prediction scheme is chosen for one of compared targets, and the compared targets are the clustering prediction scheme [25], the unpredicted scheme that admits call request immediately if there are enough bandwidths left, the proposed CAC scheme, the guard channel policy that preserves one bandwidth unit beforehand, and the guard policy that preserves four bandwidth units in advance, respectively.

For a simple case, Figure 5 illustrates that the guard channel policy with 4 bandwidth units preserved maintains the lowest CDP. On the contrary, Figure 6 shows that the guard channel policy with 4 bandwidth units preserved has the highest CBP. Although the cluster prediction scheme maintains lower CDP than guard channel schemes and the unpredicted one, it cannot keep the CDP below the predefined thresholdCDP. On the contrary, the proposed CAC scheme has maintained the CDP below the predefined thresholdCDP (0.3) since the correct prediction results is provided at time 00:09, and the corresponding CBP is almost the same as the unpredicted scheme. Although the guard channel policy with one bandwidth unit preserved sometimes can maintain the CDP below the predefined thresholdCDP, the CDPs become higher than thresholdCDP when the system utilization is under heavy load, such as time 00:13 and 00:17. Therefore, the proposed CAC with the prediction result is able to maintain the CDPs below the predefined threshold and keeps lower CBPs for simple case simultaneously.

In term of the time series data for occupation case, the system utilization has been increased 5\% since time 00:09, and it will cause increasing in CDPs and CBDs when the system utilizations are approaching to full load. Figure 7 shows that only both the proposed CAC scheme and the guard channel policy with four bandwidth units preserved can maintain the CDPs below the predefined threshold no matter what the system utilizations are, but the corresponding CBP comparisons, as shown in Figure 8, shows that the CDBs of the guard channel with four channel preserved are

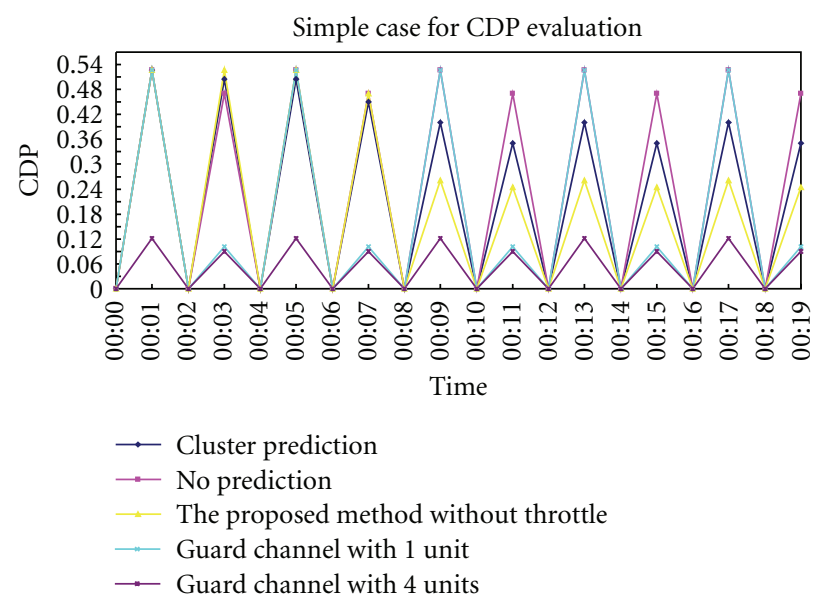

FIGURE 5: Comparison of CDPs among the proposed method and others for simple case.

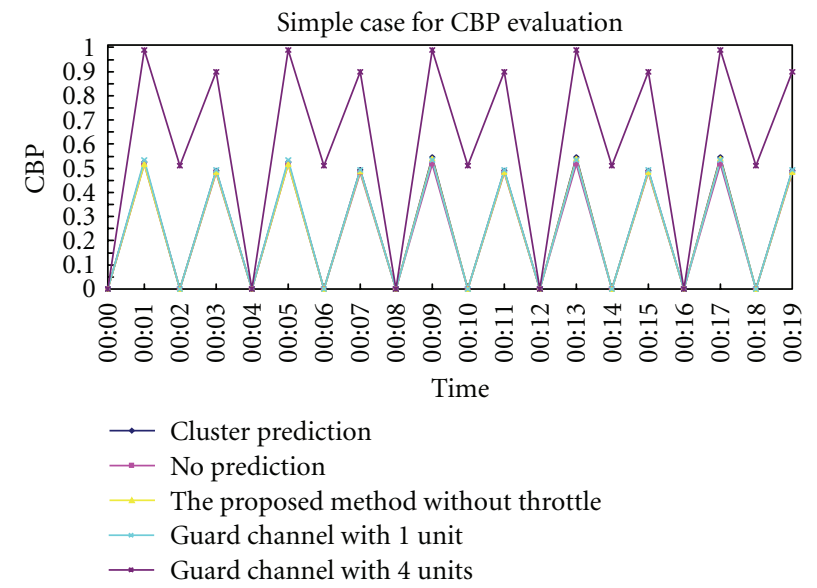

Figure 6: Comparison of CBPs among the proposed method and others for simple case.

approaching to $100 \%$ when the system utilizations are under heavy load, and the guard channel with one bandwidth unit preserved has also the same phenomenon, such as at time 00:13 and 00:17. Although the cluster prediction scheme maintains lower CDP, it cannot keep the CDPs below the predefined thresholdCDP (0.3). The proposed CAC scheme always keeps the CDPs below the predefined threshold whatever the system utilizations are, and the corresponding CBPs are also lower than the guard channel policies. The simulation results of occupation case, as shown in Figures 7 and 8, exhibit the excellent performance of the proposed CAC scheme in high system utilizations.

4.2. Evaluations of Throttle Mechanism. In terms of evaluations, there are two simulated scenarios that are the proposed scheme without the throttle mechanism applied and the proposed scheme with the throttle mechanism applied are compared for the proposed throttle mechanism. The proposed scheme without the throttle mechanism applied is the same results that are simulated in a given cell as the previous subsection, and the proposed scheme with the 


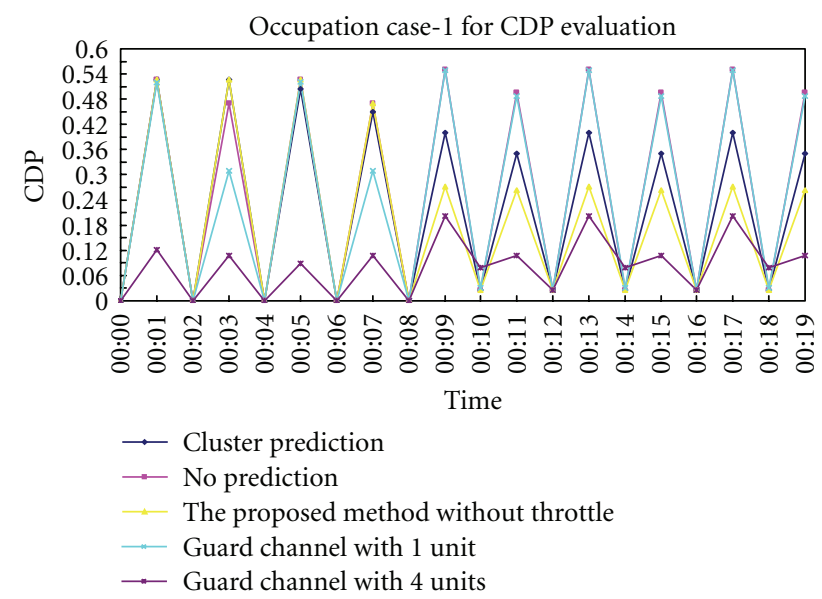

FIgURE 7: Comparison of CDPs among the proposed method and others for occupation case.

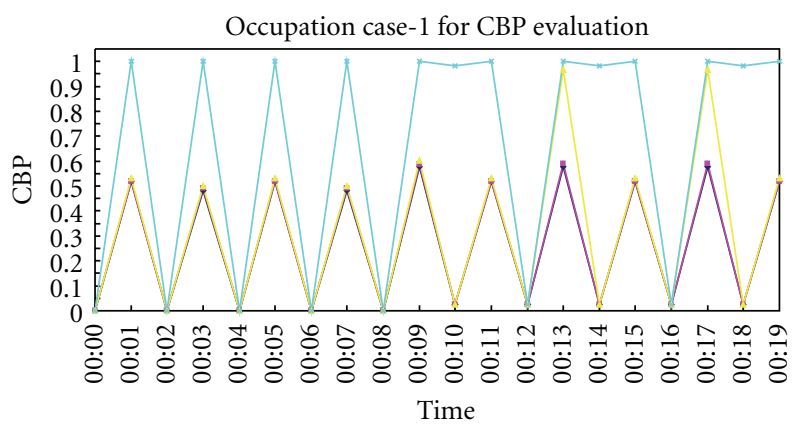

\footnotetext{
$\rightarrow$ No prediction

$\rightarrow$ The proposed method without throttle

- Guard channel with 1 unit

_- Guard channel with 4 units
}

FIGURE 8: Comparison of CBPs among the proposed method and others for occupation case-1.

throttle mechanism applied is executed for the whole service area that consists of $6 * 6$ cells. To simplify simulations, the aggregately historic data that are the CDP and the system utilization for each cell is identical. Other related input parameters are also the same as the previous subsection.

For the evaluations of throttle mechanism in simple case, Figure 9 shows that the CDP can be improved about $10 \%$ further by the proposed scheme with throttle mechanism applied, and the negative effect that is caused by the throttle mechanism on the CBP is about an increase of $6 \%$, as shown in Figure 10. Because the proposed throttle mechanism can reduce the CDP effectively with less negative effect on the CBP, it is suitable to be applied to the CAC scheme. Besides, the proposed CAC scheme with the prediction result is able to maintain the CDP below the predefined threshold and keeps lower CBP for simple case simultaneously no matter what the throttle mechanism is applied or not.

According to the simulation results, as shown in Figure 11, for the occupation case, the CDP improvement for the throttle mechanism is also approaching to $10 \%$, and the negative effect that is caused by the throttle mechanism on

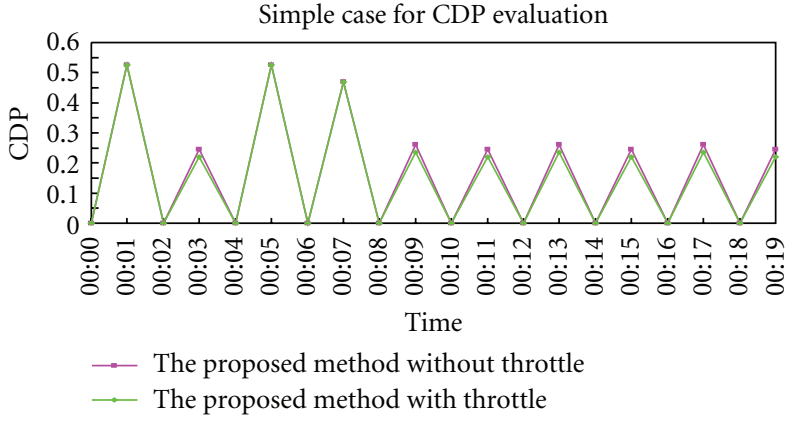

FIGURE 9: Comparison of CDPs without throttle and with throttle for simple case.

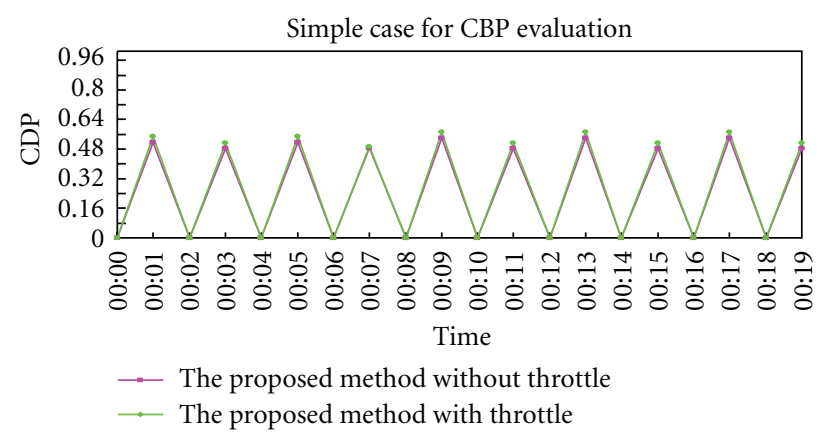

FIGURE 10: Comparison of CBPs without throttle and with throttle for simple case.

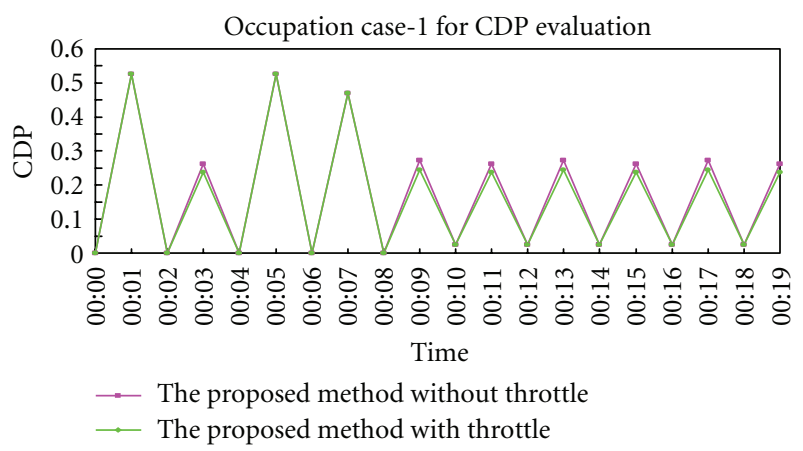

Figure 11: Comparison of CDPs without and with throttle for occupation case-1.

the CBP is the same as the simple case that is only about an increase of 6\%, as shown in Figure 12. Briefly, the proposed CAC scheme always keeps the CDP below the predefined threshold, no matter what the throttle mechanism is applied or not and whatever the system utilizations are. Moreover, the corresponding CBPs are also lower than the guard channel policies.

4.3. Discussions. In the light of the simulation results that are illustrated from Figures 5 to 12, the proposed CAC scheme not only can satisfy the predefined QoS requirement which maintains the CDP below a predefined threshold but also keeps the CBP as low as possible even though the system utilization is under heavy load. Therefore, the proposed CAC scheme can perform well, no matter what the system 


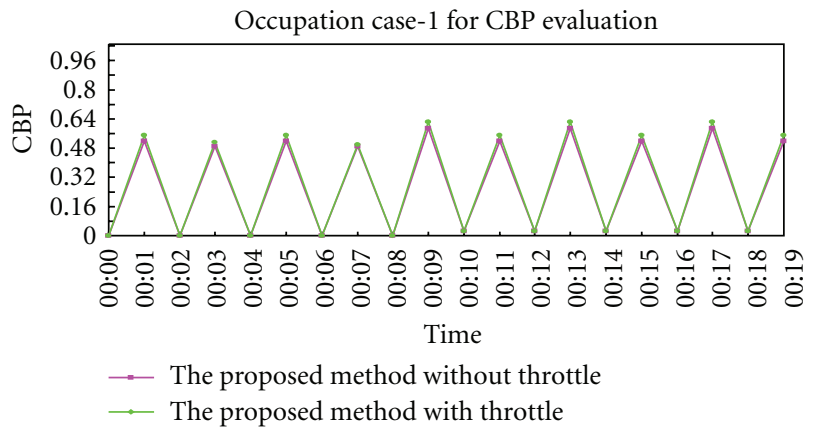

Figure 12: Comparison of CBPs without and with throttle for occupation case-1.

utilization is. Especially, the simulation results show that the proposed scheme with throttle mechanism applied and conducted under the whole service area can have excellent performances in both the CDP and the CBP simultaneously.

Although the cluster prediction scheme can also maintain lower CDP the same as the proposed one, it cannot achieve the predefined thresholdCDP. Besides, the overhead, such as time complexity and information exchange, that is caused by the cluster prediction scheme is also higher than the proposed one. Summarily, the proposed scheme is superior to the cluster prediction scheme in terms of performance and overhead.

\section{Conclusion}

An agent that consists of a prediction module and a CAC algorithm for each cell is proposed in this paper. According to the simulation results, the proposed CAC algorithm applying the prediction result of the prediction module can achieve better performance in both CDP and CBP. The prediction module can use a scalable time scale, such as minute, hour, day, week, and year of time series, to be applied in a variety of fields. For avoiding the new call request to be blocked in adjacent cells if handoff is needed, the throttle mechanism that is proposed and combined with the proposed CAC scheme participates in deciding whether to admit a new call request or not. The simulation results of the CAC algorithm also show that the proposed CAC scheme is able to meet the predefined CDP of QoS, and the CBP is less degraded than that of the cluster prediction and the traditional guard channel policies.

\section{Acknowledgment}

This paper was supported in part by the National Science Council of Taiwan under Grant NSC97-2221-E-412-001.

\section{References}

[1] W. M. P. Chaudhury and S. Onoe, "3GPP proposal for IMT2000," IEEE Communications Magazine, vol. 37, no. 12, pp. 72 $81,1999$.

[2] E. Dahlman, B. Gudmundson, M. Nilsson, and J. Skold, "UMTS/IMT-2000 based on wideband CDMA," IEEE Communications Magazine, vol. 36, no. 9, pp. 70-80, 1998.
[3] S. Marwaha, J. Indulska, and M. Portmann, "Challenges and recent advances in QoS provisioning in wireless mesh networks," in Proceedings of the IEEE 8th International Conference on Computer and Information Technology (CIT '08), pp. 618623, July 2008.

[4] G. Resta and P. Santi, "WiQoSM: an integrated QoS-aware mobility and user behavior model for wireless data networks," IEEE Transactions on Mobile Computing, vol. 7, no. 2, pp. 187198, 2008.

[5] S. H. Kim, Y. J. Kim, J. M. Moon, and M. Y. Yun, "Historybased adaptive QoS provisioning in mobile IP networks," in Proceedings of the IEEE Global Telecommunications Conference (GLOBECOM '03), vol. 6, pp. 3483-3487, December 2003.

[6] S. T. Liang and W. H. Sheng, "The mold mobility model for mobile wireless networks," in Proceedings of the 1st International Conference on Wireless Internet (WICON '05), pp. 79-84, July 2005.

[7] B. L. Mark and Z. R. Zaidi, "Mobility estimation for wireless networks based on an autoregressive model," in Proceedings of the IEEE Global Telecommunications Conference (GLOBECOM '04), vol. 6, pp. 3405-3409, December 2004.

[8] J. Sauvola and J.-Z. Sun, "Mobility and mobility management: a conceptual framework," in Proceedings of the 10th IEEE International Conference on Networks (ICON '02), pp. 205210, 2002.

[9] A. Gurijala and C. Molina, "Defining and monitoring QOS metrics in the next generation wireless networks," in Proceedings of the IEE Telecommunications Quality of Services: The Business of Success, pp. 37-42, 2004.

[10] M. Sanabani, S. Shamala, M. Othman, and Z. Zukarnain, "Adaptive resource allocation scheme based on call admission control and mobility prediction for multimedia services in wireless cellular networks," in Proceedings of the International Conference on Computer and Communication Engineering (ICCCE '08), pp. 698-701, Kuala Lumpur, Malaysia, May 2008.

[11] K. A. Agha and L. Perato, "Handover prediction: user approach versus cell approach," in Proceedings of the 4th Mobile and Wireless Communications Network International Workshop, pp. 9-11, 2002.

[12] Y. C. Kim, Y. S. Kim, B. J. Lee, and D. E. Lee, "Dynamic channel reservation based on mobility in wireless ATM networks," IEEE Communications Magazine, vol. 37, no. 11, pp. 47-51, 1999.

[13] K. Imamura and J. Tajima, "A strategy for flexible channel assignment in mobile communication systems," IEEE Transactions on Vehicular Technology, vol. 37, no. 2, pp. 92-103, 1988.

[14] I. F. Akyildiz and W. Wang, "The predictive user mobility profile framework for wireless multimedia networks," IEEE/ACM Transactions on Networking, vol. 12, no. 6, pp. 1021-1035, 2004.

[15] L. T. Lee, C. F. Wu, D. F. Tao, and K. Y. Liu, "A cell-based call admission control policy with time series prediction and throttling mechanism for supporting QoS in wireless cellular networks," in Proceedings of the IEEE International Symposium on Communications and Information Technologies (ISCIT '06), pp. 88-93, Bangkok, Thailand, October 2006.

[16] S. Wei and W. William, Time Series Analysis: Univariate and Multivariate Methods, Addison Wesley Publication, 1999.

[17] J. Heikkonen, T. Koskela, K. Kaski, and M. Varsta, "Time series prediction using recurrent SOM with local linear models," Research Report B15, Helsinki University of Technology, Laboratory of Computational Engineering, Aalto, Finland, 1997. 
[18] S. Kamitsuji and R. Shibata, "Effectiveness of stochastic neural network for prediction of fall or rise of TOPIX," Research Paper, Keio University, 2004.

[19] N. Kasabov, Evolving Connectionist Systems, Springer, London, UK, 2003.

[20] F. Haron, C. H. Yong, and O. B. Yaik, "Time series prediction using adaptive association rules," in Proceedings of the 1st International Conference on Distributed Frameworks for Multimedia Applications (DFMA '05), pp. 310-314, February 2005.

[21] M. Cornefjord, P.-O. Gaasvik, and V. Svensson, "Different methods of giving priority to handoff traffic in a mobile telephone system with directed retry," in Proceedings of the 41st IEEE Vehicular Technology Conference "Gateway to the Future Technology in Motion”, pp. 549-553, 1991.

[22] C. Bisdikian, Y. Choi, T. Kwon, and M. Naghshineh, "Call admission control for adaptive multimedia in wireless/mobile networks," in Proceedings of the IEEE Wireless Communications and Networking Conference, vol. 2, pp. 540-544, 1999.

[23] J. Hou, S. Papavassiliou, and J. Yang, "Integration of pricing with call admission control to meet QoS requirements in cellular networks," IEEE Transactions on Parallel and Distributed Systems, vol. 13, no. 9, pp. 898-910, 2002.

[24] L. T. Lee, C. F. Wu, C. Y. Tseng, and K. Y. Liu, "A dynamic reservation and call admission control policy with HMM for multimedia cellular networks," in Proceedings of the IEEE International Symposium on Communications and Information Technologies (ISCIT '06), pp. 82-87, Bangkok, Thailand, October 2006.

[25] R. Acharya and J. Tsiligaridis, "A clustering prediction scheme for wireless cellular network," in Proceedings of the International Symposium on Collaborative Technologies and Systems, pp. 298-304, St Louis, Mo, USA, May 2005.

[26] B. M. Epstein and M. Schwartz, "Predictive QoS-based admission control for multiclass traffic in cellular wireless networks," IEEE Journal on Selected Areas in Communications, vol. 18, no. 3, pp. 523-534, 2000.

[27] T. Zhang, E. Van Den Berg, J. Chennikara, P. Agrawal, J. C. Chen, and T. Kodama, "Local predictive resource reservation for handoff in multimedia wireless IP networks," IEEE Journal on Selected Areas in Communications, vol. 19, no. 10, pp. 19311941, 2001.

[28] P. Agrawal, S. Kishore, P. Ramanathan, and K. M. Sivalingam, "Dynamic resource allocation schemes during handoff for mobile multimedia wireless networks," IEEE Journal on Selected Areas in Communications, vol. 17, no. 7, pp. 1270$1283,1999$. 

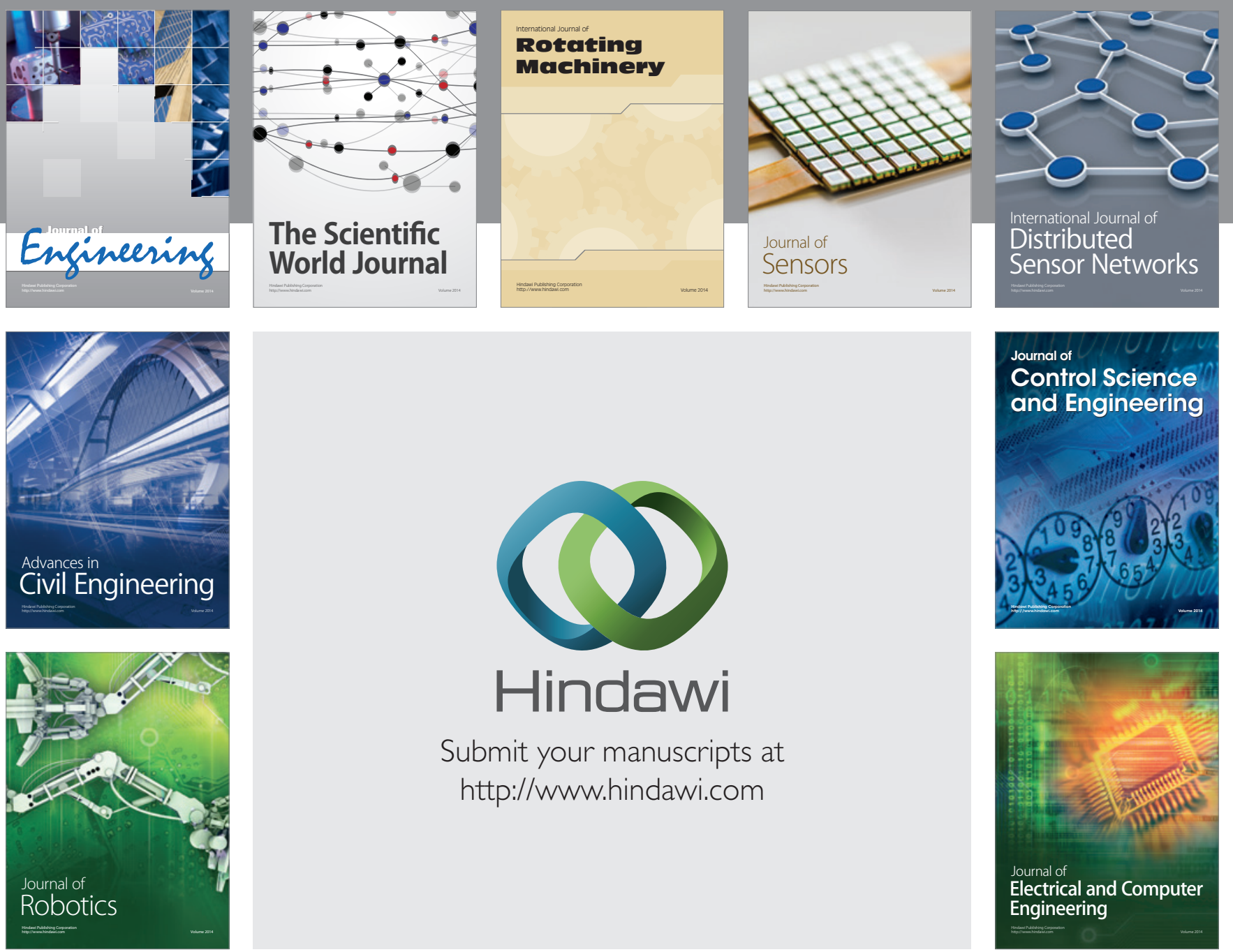

Submit your manuscripts at

http://www.hindawi.com
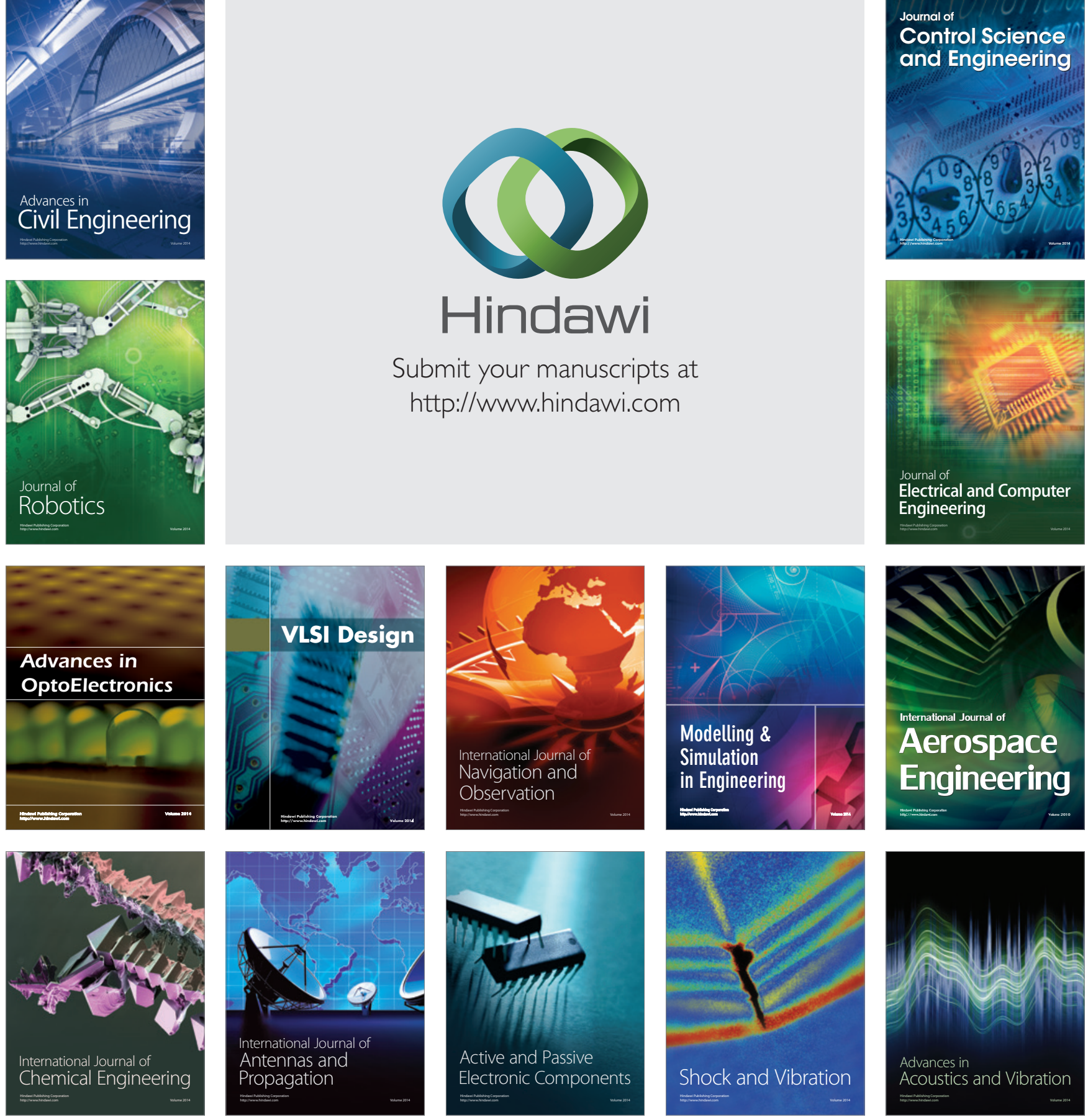